\title{
Ellipsoid movement in ideal or viscous fluid
}

\author{
Alexander Petrov ${ }^{1,2, *}$ and Mariana Lopushanski ${ }^{2, * *}$ \\ ${ }^{1}$ Institute for Problems in Mechanics RAS, Pr. Vernadskogo 101-1, 119526 Moscow, Russia \\ ${ }^{2}$ Moscow Institute of Physics and Technology, Institutskiy per. 9, 141700 Dolgoprudny, Russia
}

\begin{abstract}
The movement problem of the triaxial ellipsoid in ideal and viscous fluid in Stokes approximation is considered. With the help of the Newton potential theory it is shown that the complete solution of this problem may be expressed through a single function of two variables, which, in its turn, can be expressed through two well-known elliptic functions $-E(x, y)$ and $F(x, y)$. The result obtained simplifies the analysis of fluid velocity field when a triaxial ellipsoid is moving in it. The solution of the external boundary problem for the Stokes equations of viscous fluid flow is built. The ellipsoid in incident flow with velocity $v$ experiences the same resistance as a ball of a certain radius. Using this, the triaxial ellipsoid which experiences minimum resistance is found, and its resistance is compared to the one of a sphere.
\end{abstract}

\section{Introduction}

The solution of the movement problem of the triaxial ellipsoid $x_{1}^{2} / a_{1}^{2}+x_{2}^{2} / a_{2}^{2}+x_{3}^{2} / a_{3}^{2}=1$ in ideal and viscous fluid in Stokes approximation is constructed using the approximation of four functions which depend on four variables $[1,2]$

$$
\begin{gathered}
F_{i}\left(a_{1}, a_{2}, a_{3}, \lambda\right)=\int_{\lambda}^{\infty} \frac{a_{1} a_{2} a_{3} d u}{\left(a_{i}^{2}+u\right) \sqrt{\left(a_{1}^{2}+u\right)\left(a_{2}^{2}+u\right)\left(a_{3}^{2}+u\right)}}, \\
F\left(a_{1}, a_{2}, a_{3}, \lambda\right)=\int_{\lambda}^{\infty} \frac{a_{1} a_{2} a_{3} d u}{\sqrt{\left(a_{1}^{2}+u\right)\left(a_{2}^{2}+u\right)\left(a_{3}^{2}+u\right)}} .
\end{gathered}
$$

With the help of the Newton potential theory [3,4] it is shown that the complete solution of this problem may be expressed through a single function of two variables

$$
A_{0}(X, Y)=X Y \int_{0}^{\infty} \frac{d t}{(1+t) \sqrt{(1+t)\left(X^{2}+t\right)\left(Y^{2}+t\right)}}
$$

It may be expressed through two well-known elliptic functions

$$
E(\varphi, m)=\int_{0}^{\varphi}\left(1-m \sin ^{2} \varphi^{\prime}\right)^{1 / 2} d \varphi^{\prime}, \quad F(\varphi, m)=\int_{0}^{\varphi}\left(1-m \sin ^{2} \varphi^{\prime}\right)^{-1 / 2} d \varphi^{\prime}
$$

\footnotetext{
*e-mail: petrovipmech@gmail.com

**e-mail: masha.alexandra@gmail.com
} 
as follows

$$
A_{0}(X, Y)=\frac{2 X Y}{\left(1-X^{2}\right) \sqrt{1-Y^{2}}}\left[F\left(\arccos Y, \frac{1-X^{2}}{1-Y^{2}}\right)-E\left(\arccos Y, \frac{1-X^{2}}{1-Y^{2}}\right)\right]
$$

In this paper we strive to give an analytic solution for the ellipsoid movement in ideal and viscous fluid. In [5] a numerical solution is analyzed. The result obtained simplifies the analysis of fluid velocity field when a triaxial ellipsoid is moving in it. It also simplifies the calculus of added masses, viscous resistance and other hydrodynamical characteristics.

The result is useful for studying ellipsoidal equilibrium figures of gravitating masses.

\section{Standart equations}

Let one express functions (1) through function (3). In the first integral of (1) let us substitute the variable $u=\lambda+u^{\prime}$

$$
F_{1}\left(a_{1}, a_{2}, a_{3}, \lambda\right)=\int_{0}^{\infty} \frac{a_{1} a_{2} a_{3} d u^{\prime}}{\left(\tilde{a}_{1}^{2}+u^{\prime}\right) \sqrt{\left(\tilde{a}_{1}^{2}+u^{\prime}\right)\left(\tilde{a}_{2}^{2}+u^{\prime}\right)\left(\tilde{a}_{3}^{2}+u^{\prime}\right)}}, \quad \tilde{a}_{i}^{2}=a_{i}^{2}+\lambda, \quad i=1,2,3 .
$$

This equality is rewritten as follows

$$
F_{1}\left(a_{1}, a_{2}, a_{3}, \lambda\right)=\frac{a_{1} a_{2} a_{3}}{\tilde{a}_{1} \tilde{a}_{2} \tilde{a}_{3}} F_{1}\left(\tilde{a}_{1}, \tilde{a}_{2}, \tilde{a}_{3}, 0\right)
$$

Substituting $u^{\prime}=\tilde{a}_{1}^{2} t$ in the integral for $F_{1}\left(\tilde{a}_{1}, \tilde{a}_{2}, \tilde{a}_{3}, 0\right)$, the following equality is obtained

$$
F_{1}\left(\tilde{a}_{1}, \tilde{a}_{2}, \tilde{a}_{3}, 0\right)=A_{0}\left(\frac{\tilde{a}_{2}}{\tilde{a}_{1}}, \frac{\tilde{a}_{3}}{\tilde{a}_{1}}\right) .
$$

Thus the first function (1) is expressed through function (3) as follows

$$
F_{1}\left(a_{1}, a_{2}, a_{3}, \lambda\right)=\frac{a_{1} a_{2} a_{3}}{\tilde{a}_{1} \tilde{a}_{2} \tilde{a}_{3}} A_{0}\left(\frac{\tilde{a}_{2}}{\tilde{a}_{1}}, \frac{\tilde{a}_{3}}{\tilde{a}_{1}}\right), \quad \tilde{a}_{i}^{2}=a_{i}^{2}+\lambda, \quad i=1,2,3 .
$$

For the rest of the functions $F_{2}$ and $F_{3}$ the formulas are obtained by circular permutation of indexes.

Analogically, the formula for the function $F$ from (2) is obtained

$$
\begin{gathered}
F\left(a_{1}, a_{2}, a_{3}, \lambda\right)=\frac{a_{1} a_{2} a_{3}}{\tilde{a}_{1} \tilde{a}_{2} \tilde{a}_{3}} F\left(\tilde{a}_{1}, \tilde{a}_{2}, \tilde{a}_{3}, 0\right), \\
F\left(\tilde{a}_{1}, \tilde{a}_{2}, \tilde{a}_{3}, 0\right)=\tilde{a}_{1}^{2} B_{0}\left(\frac{\tilde{a}_{2}}{\tilde{a}_{1}}, \frac{\tilde{a}_{3}}{\tilde{a}_{1}}\right)=\tilde{a}_{2}^{2} B_{0}\left(\frac{\tilde{a}_{3}}{\tilde{a}_{2}}, \frac{\tilde{a}_{1}}{\tilde{a}_{2}}\right)=\tilde{a}_{3}^{2} B_{0}\left(\frac{\tilde{a}_{1}}{\tilde{a}_{3}}, \frac{\tilde{a}_{2}}{\tilde{a}_{3}}\right)
\end{gathered}
$$

Here function $B_{0}(X, Y)$ is defined as

$$
B_{0}(X, Y)=X Y \int_{0}^{\infty} \frac{d t}{\sqrt{(1+t)\left(X^{2}+t\right)\left(Y^{2}+t\right)}}
$$

It is expressed through the elliptic function as follows

$$
B_{0}(X, Y)=\frac{2 X Y}{\sqrt{1-Y^{2}}} F\left(\arccos Y, \frac{1-X^{2}}{1-Y^{2}}\right)
$$

For functions $A_{0}(X, Y)$ and $B_{0}(X, Y)$ the following useful properties will be proved

$$
\begin{aligned}
& A_{0}(X, Y)+A_{0}\left(\frac{1}{X}, \frac{Y}{X}\right)+A_{0}\left(\frac{1}{Y}, \frac{X}{Y}\right)=2, \\
& A_{0}(X, Y)+X^{2} A_{0}\left(\frac{1}{X}, \frac{Y}{X}\right)+Y^{2} A_{0}\left(\frac{1}{Y}, \frac{X}{Y}\right)=B_{0}(X, Y), \\
& B_{0}(X, Y)=X^{2} B_{0}\left(\frac{1}{X}, \frac{Y}{X}\right)=Y^{2} B_{0}\left(\frac{1}{Y}, \frac{X}{Y}\right) .
\end{aligned}
$$


This follows from the Newton potential theory, as presented in $[3,4]$. The proof is given in Additional Results.

Thus, all the initial functions $F_{i}\left(a_{1}, a_{2}, a_{3}, \lambda\right), F\left(a_{1}, a_{2}, a_{3}, \lambda\right)$ can be expressed through a single function (4), which we needed to prove. The applications of the formulas obtained are given in the examples below.

\section{Movement of ellipsoid in ideal fluid}

As the ellipsoid moves parallel to axis $x_{1}$, the potential of the fluid velocity field has the following form [1]

$$
\Phi_{1}=v_{\infty} x_{1} \frac{1+F_{1}\left(a_{1}, a_{2}, a_{3}, \lambda\right)}{2-F_{1}\left(a_{1}, a_{2}, a_{3}, 0\right)},
$$

where $\lambda>0$ is the solution of the equation

$$
\sum_{j=1}^{3} \frac{x_{j}^{2}}{\left(a_{j}^{2}+\lambda\right)}=1, \quad i=1,2,3,
$$

and the derivatives by coordinates $\partial \lambda / \partial x_{i}$ can be found from the following equations

$$
\frac{2 x_{i}}{a_{i}^{2}+\lambda}-\frac{\partial \lambda}{\partial x_{i}} \sum_{j=1}^{3} \frac{x_{j}^{2}}{\left(a_{j}^{2}+\lambda\right)^{2}}=0, \quad i=1,2,3 .
$$

The velocity field is found by derivation of potential by coordinates

$$
\frac{1}{v_{\infty}} \frac{\partial \Phi_{1}}{\partial x_{i}}=1+\frac{F_{1}\left(a_{1}, a_{2}, a_{3}, \lambda\right)+x_{1} \partial F_{1} / \partial x_{1}}{2-F_{1}\left(a_{1}, a_{2}, a_{3}, 0\right)}
$$

where definition (1) implies that

$$
\frac{\partial F_{1}}{\partial x_{1}}=\frac{\partial F_{1}}{\partial \lambda} \frac{\partial \lambda}{\partial x_{1}}=-\frac{a_{1} a_{2} a_{3}}{\tilde{a}_{1}^{3} \tilde{a}_{2} \tilde{a}_{3}} \frac{\partial \lambda}{\partial x_{1}} .
$$

With the help of these formulas the velocity components at any point is easily calculated. It will be shown how to perform the calculations on the following example.

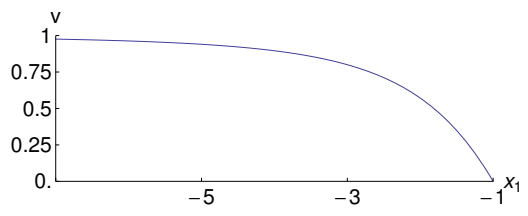

Figure 1. Dependence of dimensionless velocity $v$ on coordinate $x_{1}$.

Example 1. Build the graph of dimensionless velocity in dependence of $x_{1}$ for flow around of ellipsoid with axes $a_{1}=1, a_{2}=2, a_{3}=3$ by ideal fluid with velocity $v_{\infty}$ at infinity, in the direction of axis $x_{1}$.

Solution. For $x_{1}$ we have that $x_{1}^{2}=a_{1}^{2}+\lambda$ and with the help of Eq. (6) it is obtained that

$$
v=\frac{1}{v_{\infty}} \frac{\partial \Phi}{\partial x_{1}}=\frac{6}{\sqrt{x_{1}^{2}\left(3+x_{1}^{2}\right)\left(8+x_{1}^{2}\right)}} \frac{2-A_{0}\left(\sqrt{\frac{3+x_{1}^{2}}{x_{1}^{2}}}, \sqrt{\frac{8+x_{1}^{2}}{x_{1}^{2}}}\right)}{2-A_{0}(2,3)} .
$$


The graph of the obtained dependence of velocity $v$ on coordinate $x_{1} \in(-7,-1)$ is presented in Fig. 1.

When an ellipsoid rotates in fluid relative to axis $x_{1}$ with angular velocity $\omega_{1}$ the potential of fluid velocity field has the following form [1]

$$
C \omega_{1} x_{2} x_{3}\left(F_{3}\left(a_{1}, a_{2}, a_{3}, \lambda\right)-F_{2}\left(a_{1}, a_{2}, a_{3}, \lambda\right)\right), \quad C=\frac{a_{2}^{2}-a_{3}^{2}}{2\left(a_{2}^{2}-a_{3}^{2}\right)-\left(a_{2}^{2}+a_{3}^{2}\right)\left(F_{3}\left(a_{1}, a_{2}, a_{3}, 0\right)-F_{2}\left(a_{1}, a_{2}, a_{3}, 0\right)\right)} .
$$

The difference of functions $F_{3}-F_{2}$ is expressed with the help of formulas (6) in terms of function $A_{0}(X, Y)$ as follows

$$
F_{3}\left(a_{1}, a_{2}, a_{3}, \lambda\right)-F_{2}\left(a_{1}, a_{2}, a_{3}, \lambda\right)=\frac{a_{1} a_{2} a_{3}}{\tilde{a}_{1} \tilde{a}_{2} \tilde{a}_{3}}\left(A_{0}\left(\frac{\tilde{a}_{1}}{\tilde{a}_{3}}, \frac{\tilde{a}_{2}}{\tilde{a}_{3}}\right)-A_{0}\left(\frac{\tilde{a}_{1}}{\tilde{a}_{2}}, \frac{\tilde{a}_{3}}{\tilde{a}_{2}}\right)\right) .
$$

The attached masses $\lambda_{i}, i=1,2, \ldots, 6$ are defined as the coefficients of kinetic energy $T$ of the fluid when a triaxil ellipsoid moves in it

$$
2 T=\sum_{i=1}^{3}\left(\lambda_{i} U_{i}^{2}+\lambda_{3+i} \omega_{i}^{2}\right),
$$

where $U_{i}$ and $\omega_{i}$ are the components of solid body movement velocity and the components of its angular velocity directed along the main axes of ellipsoid.

The formulas for calculating $\lambda_{i}, i=1,2, \ldots, 6$ can be found in [1,2]. It is convenient to calculate their ratio with the ellipsoid mass, with fluid density $m$ and the moments of inertia $J_{1}, J_{2}, J_{3}$ and express them through functions $A_{0}(X, Y)$, which are calculated in Eq. (4)

$$
\begin{aligned}
& \mu_{1}=\lambda_{1} / m=\frac{A_{0}\left(a_{2} / a_{1}, a_{3} / a_{1}\right)}{2-A_{0}\left(a_{2} / a_{1}, a_{3} / a_{1}\right)}, \\
& \mu_{4}=\lambda_{4} / J_{1}=\frac{\left(a_{2}^{2}-a_{3}^{2}\right)^{2} /\left(a_{2}^{2}+a_{3}^{2}\right)}{2\left(a_{2}^{2}-a_{3}^{2}\right)-\left(a_{2}^{2}+a_{3}^{2}\right) A_{123}} A_{123}, \\
& A_{123}=A_{0}\left(a_{1} / a_{3}, a_{2} / a_{3}\right)-A_{0}\left(a_{3} / a_{2}, a_{1} / a_{2}\right), \\
& m=\frac{4 \pi \rho a_{1} a_{2} a_{3}}{3}, \quad J_{1}=\frac{4 \pi \rho a_{1} a_{2} a_{3}}{15}\left(a_{2}^{2}+a_{3}^{2}\right) .
\end{aligned}
$$

The coefficients $\mu_{2}, \mu_{3}$ can be obtained from the formula for $\mu_{1}$ by circular permutation of indexes. Analogically, may be obtained the formulas for coefficients $\mu_{5}, \mu_{6}$ from the formula for $\mu_{4}$.

Example 2. Find the numerical values of $\mu_{1}, \mu_{2}, \ldots, \mu_{6}$ for ellipsoid with axes $a_{1}=1, a_{2}=$ $2, a_{3}=3$.

Solution. For values $a_{1}=1, a_{2}=2, a_{3}=3$ with the help of formulas (5) and (4) we calculate three values

$$
\begin{gathered}
A_{0}\left(a_{2} / a_{1}, a_{3} / a_{1}\right)=A_{0}(2,3)=1.153, A_{0}\left(a_{3} / a_{2}, a_{1} / a_{2}\right)=A_{0}(3 / 2,1 / 2)=0.534, \\
A_{0}\left(a_{1} / a_{3}, a_{2} / a_{3}\right)=A_{0}(1 / 3,2 / 3)=0.312
\end{gathered}
$$

With the help of these values, by the third formula from Eq. (12) it is found that

$$
\begin{aligned}
A_{123} & =0.312-0.534=-0.221 \\
A_{231}=1.153-0.312 & =0.840, \quad A_{312}=0.534-1.153=-0.619,
\end{aligned}
$$

and from the first and second formulas from Eq. (12) the coefficients

$$
\mu_{1}=1.361, \quad \mu_{2}=0.364, \quad \mu_{3}=0.185, \quad \mu_{4}=0.0599, \quad \mu_{5}=0.708, \quad \mu_{6}=0.383
$$

are calculated.

Example 3. Find the pressure distribution on ellipsoid surface in incident flow with velocity $v_{\infty}$, directed along axis $x_{1}$. 
Solution. On the ellipsoid surface the normal derivative of potential (11) equals zero, thus the fluid velocity on surface equals the derivative by tangential direction to the surface of potential $s$

$$
\begin{gathered}
\left.\Phi_{1}\right|_{\lambda=0}=v_{\infty} x_{1}\left(1+\frac{F_{1}\left(a_{1}, a_{2}, a_{3}, 0\right)}{2-F_{1}\left(a_{1}, a_{2}, a_{3}, 0\right)}\right)=v_{\infty} x_{1}\left(1+\mu_{1}\right), \\
v=\frac{\partial \Phi_{1}}{\partial s}=v_{\infty}\left(1+\mu_{1}\right) \frac{\partial x_{1}}{\partial s} .
\end{gathered}
$$

The derivative by coordinate of $s$ may be expressed through the projection of the normal on axis $x_{1}$ with the help of equality

$$
n_{1}^{2}+\left(\frac{\partial x_{1}}{\partial s}\right)^{2}=1
$$

This and formulas (13) imply that

$$
\frac{v^{2}}{v_{\infty}^{2}}=\left(1+\mu_{1}\right)^{2}\left(1-n_{1}^{2}\right)
$$

By the Bernulli Theorem one may obtain that the pressure coefficient $c_{p}$ is

$$
c_{p}=\frac{2\left(p-p_{\infty}\right)}{\rho v_{\infty}^{2}}=1-\frac{v^{2}}{v_{\infty}^{2}}=1-\left(1+\mu_{1}\right)^{2}\left(1-n_{1}^{2}\right) .
$$

Thus the solution was built.

An analogy between the law for the pressure coefficient obtained and Newton's formula $c_{p}=2 n_{1}^{2}$ may be drawn. Newton supposed that the particles in fluid experience non-elastic collisions with the body surface [6]. The Newton formula is valid for body resistance coefficient in hypersonic flow and is used to calculate the body forms of smallest resistance [7]. In both formulas the pressure coefficient depends only on the element orientation with respect to the incident flow velocity.

\section{Ellipsoid in viscous fluid}

The solution of the external boundary problem for the Stokes equations of viscous fluid flow

$$
\mu \Delta \mathbf{v}=\nabla p
$$

can be built.

The ellipsoid flow around velocity field $\mathbf{v}$ with velocity $v_{\infty}$, directed along axis $x$ and sticking conditions on the ellipsoid boundary was found by Oberbeck in 1876 [1]. It may be presented as follows

$$
\begin{gathered}
\mathbf{v}=\nabla H+x_{1} \nabla G+\mathbf{i}\left(-G+v_{\infty}\right), \quad p=2 \mu \partial G / \partial x_{1}, \quad H=-k v_{\infty} x_{1} F_{1}\left(a_{1}, a_{2}, a_{3}, \lambda\right), \\
G=\frac{k v_{\infty}}{a_{1}^{2}} F\left(a_{1}, a_{2}, a_{3}, \lambda\right), \quad k=\frac{1}{A_{0}\left(\frac{a_{2}}{a_{1}}, \frac{a_{3}}{a_{1}}\right)+B_{0}\left(\frac{a_{2}}{a_{1}}, \frac{a_{3}}{a_{1}}\right)} .
\end{gathered}
$$

Formulas (6) and (7) imply that functions $F_{1}$ and $F$ may be expressed through function $A_{0}(X, Y)$.

The ellipsoid in incident flotation with velocity $v_{\infty}$ experiences the same resistance as a ball of radius

$$
\begin{gathered}
R=\frac{8 a_{2} a_{3}}{3 a_{1}\left(A_{0}(X, Y)+B_{0}(X, Y)\right)}, \\
X=a_{2} / a_{1}, \quad Y=a_{3} / a_{1} .
\end{gathered}
$$

It equals $6 \pi \mu R v_{\infty}$. It is convenient to calculate the ratio of $R$ to the radius of a sphere of equal volume $l=\left(a_{1} a_{2} a_{3}\right)^{1 / 3}$. The ratio $R / l$ shows how many times the resistance of ellipsoid 
exceeds the resistance of sphere of radius $l$. For an axially symmetric ellipsoid $a_{2}=a_{3}$ and so this ratio has the following form

$$
\frac{R}{l}=\frac{8 X^{4 / 3}}{3\left(A_{0}(X, X)+B_{0}(X, X)\right)}, \quad X=a_{2} / a_{1}
$$

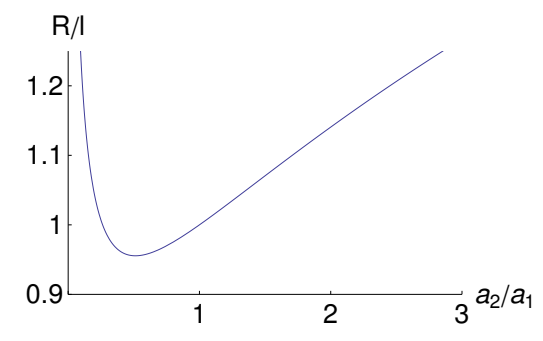

Figure 2. Dependence $R / l$ on the ratio of ellipsoid axes $a_{2} / a_{1}$.

From the dependence graph of $R / l$ in Fig. 1 it may be seen that $R / l$ has a minimum. Calculations show that $\min (R / l)=0.95551$ if $a_{2} / a_{1}=0.51226$. From this we deduce that among ellipsoids of constant volume the smallest resistance is experienced by a flattened ellipsoid with axes ratio $a_{2} / a_{1}=0.51226$. It is smaller by $4.45 \%$ than the resistance experienced by a sphere of radius $l$.

Let us show that the minimum found for axially symmetrical ellipsoids is the global minimum for triaxial ellipsoid, i.e. the function of two variables

$$
r(X, Y)=\frac{8(X Y)^{2 / 3}}{3\left(A_{0}(X, Y)+B_{0}(X, Y)\right)}, \quad X=a_{2} / a_{1}, \quad Y=a_{3} / a_{1}
$$

has a global minimum at $X=Y=0.51226$. Here the functions $A_{0}(X, Y)$ and $B_{0}(X, Y)$ are expressed through elliptic functions by formulas (4) and (9).

To prove this let us consider the following variables $X=x(1+z), Y=x(1-z)$. Symmetry by variables $X, Y$ implies that in these new variables the function $\rho(x, z)=r(x(1+z), x(1-z))$ is even by $z$ for constant values of $x$. Moreover, $\rho(x, z)>\rho(x, 0)$ for all admissible values $0<z<1$.

This fact is implied by the graphs of function $\Delta \rho(x, z)=\rho(x, z)-\rho(x, 0)+x$ for different values of $x$, given in Fig. 2. From this we obtain an inequality in initial variables $r(X, Y) \geq$ $r((X+Y) / 2,(X+Y) / 2) \geq \min r(x, x)$, which we needed to prove.

\section{Conclusion}

The analytical solution for the movement of an ellipsoid in ideal and viscous fluid was obtained. The triaxial ellipsoid which experiences minimum resistance was found, and its resistance was compared to the one of a sphere.

The study has been supported by the Russian Foundation for Basic Research (project N. 17-0100901) in MIPT.

\section{References}

[1] G. Lamb, Hydrodynamics (Gostehizdat, Moscow-Leningrad, 1947) 
[2] N. Kochin, I. Kibel, N. Rose, Theoretical Hydrodynamics. V. 1 (Fizmatgiz, Moscow, 1963)

[3] L. Sretenskii, Theory of Newton Potential (OGIZ, Moscow, 1946)

[4] S. Chandrasekhar, Ellipsoidal figures of equilibrium (Yale University Press, New Haven, 1969)

[5] E. Band, P. Payne, The Aeronautical Quaterly 31(1) (1980)

[6] I. Newton, Collected works of Krylatov, A.N. 7 (1936)

[7] G. Chernyi, Gas flow with large supersonic speed (Fizmatlit, Moscow, 1959) 